\title{
Cournot Duopoly when the Competitors Operate under Capacity Constraints
}

Tönu Puu and Anna Norin

Centre for Regional Science and Department of Economics

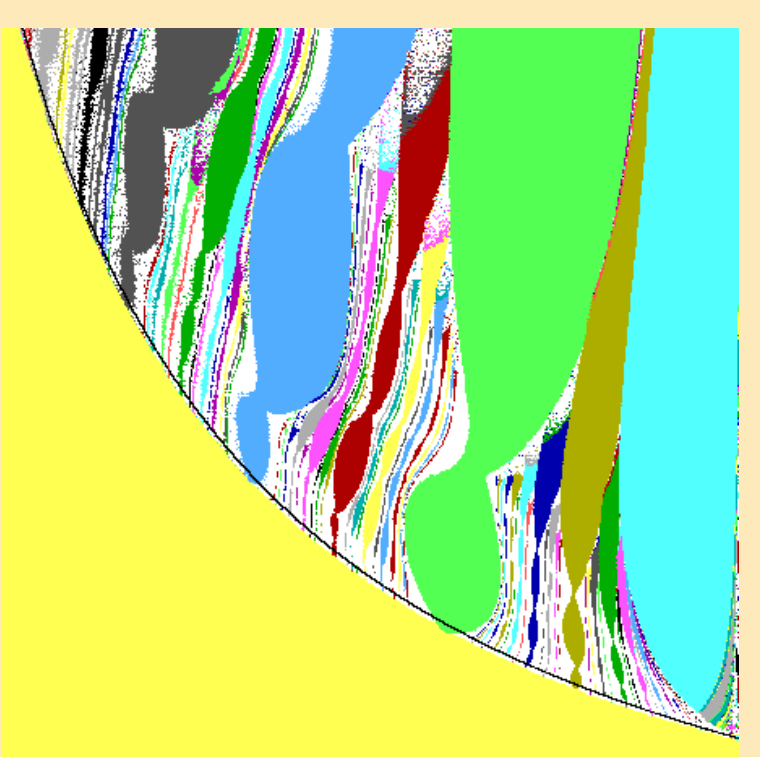





\title{
Cournot Duopoly when the Competitors Operate under Capacity Constraints
}

\author{
Tönu Puu and Anna Norin
}

Centre for Regional Science and Department of Economics

\begin{abstract}
The paper considers Cournot duopoly where the competitors have capacity constraints. An isoelastic demand function, which always results when consumers maximise utility functions of the Cobb-Douglas type, is used. It has been demonstrated that isoelastic demand, combined with constant marginal costs, results in complex dynamics. The purpose of the present paper is to reconsider the case, using in stead cost functions with capacity limits. This is a point on which Edgeworth insisted as important. Comparisons between cases of few large and many small competitors cannot be made when firms have constant returns and hence are all infinitely large in potential.
\end{abstract}

Font: Minion (Adobe)

CERUM Working Paper 50:2002

ISBN 91-7305-320-1

ISSN 1404-5362

CERUM; Umeå University; sE-90187 Umeå; Sweden

Ph.: +46-90-786.6079 Fax: +46-90-786.5121

Email: regional.science@cerum.umu.se

www.umu.se/cerum 



\section{Contents}

The Model . . . . . . . . . . . . . . . . . . . . . . . . 7

Capacity Constraints . . . . . . . . . . . . . . . 7

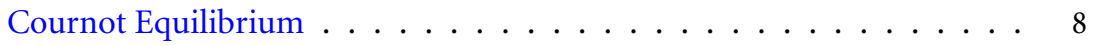

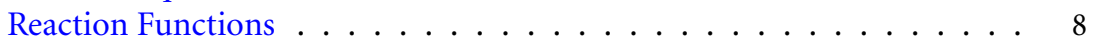

Sequential versus Simultaneous Adjustment . . . . . . . . . . . . . 9

Stability at the Cournot Point . . . . . . . . . . . . 10

The Shape of Reaction Functions . . . . . . . . . . . . . . . 11

Examples . . . . . . . . . . . . . . . . . . 12

Adaptive Adjustment . . . . . . . . . . . . . . . . . . . 15

Stability in the Adaptive Model . . . . . . . . . . . . . . . . . 16

Subcriticality and Coexistence . . . . . . . . . . . . . . 17

Periodic Solutions and Other . . . . . . . . . . . . . . . . . 18

Critical Lines and Absorbing Areas . . . . . . . . . . . . . . . 25

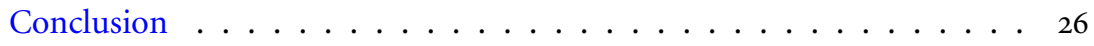

References . . . . . . . . . . . . . . . . . . . 27 



\section{The Model}

Oligopoly theory is one of the oldest branches of mathematical economics, dating back to Cournot (1838), who considered it both in terms of equilibrium and in the sense of dynamics. As in the case of so many other models in economics, the variants taught in modern text books used linear reaction functions, and concentrated on stability of equilibrium. Once nonlinear dynamics started dissipating to economics, Rand (1978) conjectured the possibility of interesting dynamics phenomena in the Cournot duopoly model when both reaction functions start at the origin, reach a maximum, and then decrease to zero again. However, he gave no subject matter rationale in terms of economics for this shape.

In Puu (1991), one of the present authors suggested an isoelastic demand function for a Cournot duopoly. The inverse of this demand function reads:

$$
p:=\frac{1}{x+y}
$$

where $p$ denotes commodity price, and $x$ and $y$ denote the supplies of the two competitors. It was shown that this demand function, combined with constant marginal production costs, actually resulted in the type of reaction functions suggested by Rand. Dana and Montrucchio (1986) considered it unlikely that the reaction functions should start at the origin. By way of example they suggested a similar, though more complicated function than (1), which would avoid this problem.

The function in (1), however, arises in a natural way in standard consumption theory, whenever consumers maximize utility functions of the Cobb-Douglas type. The reaction functions can be solved for in closed form, and, in fact, as was shown in Puu (1991), there is a period doubling cascade to chaos.

This framework with constant marginal costs does not take note of an argument on which Edgeworth (1897) insisted, concerning the importance of limited supply capacities for the firms. It is true that Edgeworth considered Bertrand duopoly, rather than Cournot duopoly, but it is equally interesting to consider capacity limits in the latter case. In the above setting, long standing issues, such as whether an increase of the number of competing firms stabilizes or destabilizes Cournot equilibria, cannot be settled, because any firm with constant marginal costs, has in principle infinite supply capacity. There can hence be no comparison of cases with few big and many small firms when there are no capacity limits. A favorite idea among economists had been that there is a smooth way leading from monopoly, over oligopoly, to perfect competition, the Cournot point becoming a competitive equilibrium in the limit when the number of competitors goes to infinity. This was challenged by Agiza (1998), and Ahmed and Agiza (1998), who showed that, using the original Puu (1991) model, an increasing number of competitors could, on the contrary, destabilize equilibrium. It would be most interesting to know the answer if one skips the assumption of unlimited capacity which was implicit in the original setting.

\section{Capacity Constraints}

A first step to this end would be to introduce capacity limits in the model. So, suppose the two firms have the following total production cost functions:

$$
\begin{aligned}
& T C_{1}(x):=-\ln \left(1-\frac{x}{u}\right) \\
& T C_{2}(y):=-\ln \left(1-\frac{y}{v}\right)
\end{aligned}
$$

where $u$ and $v$ denote the capacity limits. These functions start with zero cost at zero production and go to infinity when the capacity limits are approached. The 
corresponding marginal costs are:

$$
\begin{aligned}
M C_{1}(x) & =\frac{d}{d x} T C_{1}(x)=\frac{1}{u-x} \\
M C_{2}(y) & =\frac{d}{d y} T C_{2}(y)=\frac{1}{v-y}
\end{aligned}
$$

On the other hand marginal revenues are easily calculated from (1), as:

$$
\begin{aligned}
M R_{1}(x, y) & =\frac{\partial}{\partial x}(p x)=\frac{y}{(x+y)^{2}} \\
M R_{2}(x, y) & =\frac{\partial}{\partial y}(p y)=\frac{x}{(x+y)^{2}}
\end{aligned}
$$

\section{Cournot Equilibrium}

Equating $M R_{i}=M C_{i}$, gives two handy equations:

$$
\begin{aligned}
& \frac{y}{(x+y)^{2}}=\frac{1}{u-x} \\
& \frac{x}{(x+y)^{2}}=\frac{1}{v-y}
\end{aligned}
$$

Dividing these equations, and rearranging, we find that $x / u=y / v$, which, substituted back in equations (5), gives us closed form expressions for $x, y$ in terms of $u, v$ :

$$
\begin{aligned}
\bar{x} & =\frac{u^{2} v}{u^{2}+3 u v+v^{2}} \\
\bar{y} & =\frac{u v^{2}}{u^{2}+3 u v+v^{2}}
\end{aligned}
$$

These are the coordinates of the Cournot point where the reaction curves for the two competitors intersect.

\section{Reaction Functions}

These curves can themselves be derived from (5) by solving the first of them for $x$, the second for $y$ :

$$
\begin{aligned}
x^{\prime} & =f(y):=\frac{1}{2} \sqrt{4 u y+5 y^{2}}-\frac{3}{2} y \\
y^{\prime} & =g(x):=\frac{1}{2} \sqrt{4 v x+5 x^{2}}-\frac{3}{2} x
\end{aligned}
$$

It is trivial to check that the appropriate second order conditions for profit maxima are always fulfilled. We used the convenient practice, which more and more is becoming standard, to denote the next iterate of a map by a dash. If the variables without dashes on the right refer to the values they take in time period $t$, then the variables with dashes on the left refer to their values in the next time period, $t+1$. In this way we can avoid the messy indexing of the variables as has been traditional in the economics literature using difference equations. Any confusion is avoided if we decide to never use the dash as a symbol for a derivative. There are several alternative notations available for these.

The system ( 7 ) is written as a dynamical system, whereas (5) had the look of a simultaneous system of equations. We regain the latter by just putting $x^{\prime}=x=\bar{x}$, $y^{\prime}=y=\bar{y}$ in (7), and rearranging. 
Next note the particularity that according to (7) the outputs, $x$ or $y$ respectively, become negative whenever $y>u$ or $x>v$. This is due to the fact that, whenever the competitor supplies more than the capacity limit for a firm itself, then its own profit function will be negative for all positive outputs, and positive only for negative outputs, including a spurious profit maximum at a certain negative output. This being nonsense we have to rule it out by reformulating the reaction functions as:

$$
\begin{aligned}
x^{\prime} & =F(y):=\left\{\begin{array}{cc}
f(x) & y \leq u \\
0 & y>u
\end{array}\right. \\
y^{\prime} & =G(x):=\left\{\begin{array}{cc}
g(y) & x \leq v \\
0 & x>v
\end{array}\right.
\end{aligned}
$$

where $f(x), g(y)$ are as defined in (7) above. This formulation of the map takes care of the aforementioned absurdity with firms producing negative outputs. It also is a means to avoid problems in simulation using the map iteratively. Without the nonzero clause, the numerical calculation of iterates could break down any moment the computer would try to calculate square roots of negative numbers.

It should, however, be noted that at any live simulation we would have to replace the exact zero by some arbitrarily small positive number. The reason is that the reaction curves (8), except at the Cournot equilibrium, also intersect at the origin, as we can easily check. The origin is an always unstable fixed point, and no process would ever stay there under any perturbation. The computer would, however, stick there, unless we use some means to keep the process at some distance from the exact origin.

The cases where the zero branches of the reaction functions are visited have been studied quite early, by Palander (1939). The logic of these cases is that whenever one firm supplies too much, then the best reply by the competitor is to produce nothing. The remaining firm then becomes a monopolist and reduces its supply accordingly. At this stage, however, it becomes profitable for the other firm to reenter, and there results one period of genuine duopoly, after which the whole starts all over again. This is a recurrent sequence of events, and it can also happen that both firms throw each other out by taking turns. This was all described by Palander, though in a different setting than the present.

\section{Sequential versus Simultaneous Adjustment}

Palander, like other of his contemporaries, put much emphasis on how the firms adjusted according to (7) or (8), sequentially or simultaneously. It was assumed as a matter of course that it would make a big difference whether both firms adjusted at once from the competitor's last supply, or if they took turns.

As a matter of fact there is no essential difference at all, as we can see by the following argument. By substituting from one into the other of the reaction functions (7) or (8) we obtain two essentially independent iterations

$$
\begin{aligned}
& x^{\prime \prime}=F \circ G(x) \\
& y^{\prime \prime}=G \circ F(y)
\end{aligned}
$$

each in one variable alone. The iterations are now based on a time lag twice as long as the one originally introduced, giving the variable value at time $t+2$ when the value at $t$ is known.

In this way we only get the trajectory for every second period, but, as the difference equations are second order, we need two initial conditions for two consecutive time periods. If we have such initial conditions, then each equation of (9) produces a complete trajectory. The two processes are independent, and the trajectories are completely invariant as to what was assumed on the sequential/simultaneous adjustment issue. The only difference is the way of pairing the two time series together. There also is a difference as to the freedom of choice of initial conditions. 
The sequential case does, in fact, not allow us the full liberty of choosing two initial conditions, just one.

It can, however, happen that the system itself settles at alternative adjustment. If so, the points visited are located on the reaction functions, something which is not generally the case. Such special trajectories were given the name "Markov Perfect Equilibria" (MPE), though they result from genuine recursive dynamics and have little to do with equilibrium. See Bischi, Mammana, and Gardini (2000) and Dana and Montrucchio (1986). This can happen due to the fact that the union of the graphs of the reaction functions is an invariant set, which may be attractive with a proper basin of attraction with a nonzero area measure.

\section{Stability at the Cournot Point}

We can easily check out stability of the Cournot point. Differentiating the equations (7) we obtain:

$$
\begin{aligned}
& D f(y)=\frac{2 u+5 y}{2 \sqrt{4 u y+5 y^{2}}}-\frac{3}{2} \\
& D g(x)=\frac{2 v+5 x}{2 \sqrt{4 v x+5 x^{2}}}-\frac{3}{2}
\end{aligned}
$$

Note that $\operatorname{Lim}_{y \rightarrow 0} D f(y)=\infty$ and $\operatorname{Lim}_{x \rightarrow 0} D g(x)=\infty$. This indeed indicates that the intersection point of the reaction curves at the origin is always unstable.

As for the Cournot equilibrium point we substitute the coordinates we found in (6) into (10), which simplify to:

$$
\begin{aligned}
\operatorname{Df}(\bar{y}) & =\frac{u^{2}-v^{2}}{v(2 u+3 v)} \\
D g(\bar{x}) & =\frac{v^{2}-u^{2}}{u(3 u+2 v)}
\end{aligned}
$$

For stability of the Cournot point it is the product of the derivatives (11) which matters. Due to the chain rule, $D f(\bar{y}) D g(\bar{x})$ equals the derivative of any of the composite functions, i.e. $D f(\bar{y}) D g(\bar{x})=D(f \circ g(\bar{x}))=D(g \circ f(\bar{y}))$. Whenever this product of the derivatives:

$$
D f(\bar{y}) D g(\bar{x})=-\frac{\left(u^{2}-v^{2}\right)^{2}}{u v(2 u+3 v)(3 u+2 v)}
$$

exceeds unity in absolute value, then the Cournot point loses stability.

It is obvious that, $u, v$ being positive production capacities, $D f(\bar{y}) D g(\bar{x})$ is always nonpositive, but it can become equal to -1 , which is a point of bifurcation, a flip (or period doubling) bifurcation to be exact.

We can also check stability by writing down the Jacobian matrix at the Cournot point, using the expressions (11):

$$
J=\left[\begin{array}{cc}
0 & \frac{u^{2}-v^{2}}{v(2 u+3 v)} \\
\frac{v^{2}-u^{2}}{u(3 u+2 v)} & 0
\end{array}\right]
$$

For stability its determinant

$$
|J|=\frac{\left(u^{2}-v^{2}\right)^{2}}{u v(3 u+2 v)(2 u+3 v)}
$$

must not exceed unity in absolute value. As we see $D f(\bar{y}) D g(\bar{x})=-|J|$, so this is the same condition as before. 
To find the parameter combinations for which stability is lost, put $|J|=1$ in (14), and rearrange. Thus:

$$
u^{4}-6 u^{3} v-15 u^{2} v^{2}-6 u v^{3}+v^{4}=0
$$

which, as a matter of fact, factorizes into:

$$
(u-a v)(a u-v)(u-b v)(u b-v)=0
$$

where

$$
a, b:=\frac{1}{2}(1 \pm \sqrt{2})(3+\sqrt{13})
$$

From (16) we immediately see that $a>0$ and $b<0$. Accordingly, using $a$, the bifurcations in the $u, v$ parameter plane occur in quadrants where the parameters have equal sign, whereas, using $b$, they occur in quadrants where the parameters have opposite sign. As $u, v$ are positive measures for maximum production capacities, they are both positive, so only the first two factors of (15) are relevant for the flip bifurcations which are of factual interest to us.

As a result, there is a stability region for the Cournot equilibrium point where:

$$
\frac{1}{a}<\frac{u}{v}<a
$$

As $a \approx 7.97$, we see that, for the Cournot point to be destabilized, there must be a considerable asymmetry between the firms in terms of their capacity limits. Only when one firm is about eight times as large in terms of its maximum production capacity as the competitor does the period doubling cascade start. One might therefore consider that complex dynamics is an unlikely phenomenon.

Such a conclusion would, however, be totally mistaken. Out of an immense set of qualitatively similar cost functions with capacity bounds we chose one rare case for which closed form solutions were possible. By smooth topological modifications we could decrease the amount of asymmetry needed for the flip bifurcation to almost any extent, but then we no longer get any closed form solutions.

So, we are satisfied with having shown that complex dynamics are possible for the representative case where we can make all the derivations in simple closed form, and we need not bother too much about exact numerical ranges.

We note that the bifurcation condition (17) only depends on the capacity ratio $u / v$, not on the capacities separately. Below we will repeatedly find this feature in connection with other important facts as well. This is so because the basic iteration (7) itself can easily, using the the new variables $X:=x / u, Y:=y / v$, be written in a form which only depends on this ratio $u / v$, or its reciprocal, $v / u$, but not on the capacities themselves. For this reason we have a basic model which only has one single parameter. Inclusion of the zero branch in (8) does not change anything in this respect, neither does the introduction of adptation coeffiecients below. Further, the systems are reciprocally symmetric with respect to $u / v$ and $v / u$, so, whenever we want we may, without any loss of generality, just fix any one of the two capacities at an arbitrary value, and then study the system as the other capacity is changed.

\section{The Shape of Reaction Functions}

We have already noted two things about the reaction functions (7): i) They both start at the origin and get down to zero again once the competitor's supply equals the maximum capacity of the firm itself. After that point the expression ( 7 ) is replaced by zero according to (8). ii) They start growing with infinite slopes.

We can easily find out more. The reaction functions are smooth until the replacement points mentioned, because the derivatives (10) obviously are continuous functions. 
Further, taking the second derivatives of ( 7 ), we easily get:

$$
\begin{aligned}
& D^{2} f(y)=-\frac{2 u^{2}}{\left(4 u y+5 y^{2}\right)^{3 / 2}}<0 \\
& D^{2} g(x)=-\frac{2 v^{2}}{\left(4 v x+5 x^{2}\right)^{3 / 2}}<0
\end{aligned}
$$

Negativity holds for the whole ranges of the functions, so the reaction functions are uniformly convex from above. As convex functions they have unique maxima.

To locate these put $D f(y)=0$ and $D g(x)=0$ in (10), and solve to obtain $y=$ $u / 5$ and $x=v / 5$ respectively. As the definition ranges for $(7)$ are $y \in(0, u)$ and $x \in(0, v)$ respectively, the maxima occur at one fifth of the full ranges. Further, by substituting these values into the reaction functions ( 7 ), we immediately find that $x=y$ in the maximum points for both reaction functions, i.e. the maxima for $x$ and $y$ are:

$$
\begin{aligned}
& \frac{v}{5}=g\left(\frac{v}{5}\right) \\
& \frac{u}{5}=f\left(\frac{u}{5}\right)
\end{aligned}
$$

respectively. They are hence both located on the diagonal in the $x, y$-phase diagram.

What has been said indicates that the reaction functions indeed have the shapes assumed by Rand. However, Rand assumed both ranges to be the unit interval, whereas we saw that there must be some asymmetry between the competitors in terms of their maximum capacities. As a consequence, the definition ranges will be different. Further, when both maxima are located on the diagonal, there cannot be any multiple nonzero intersections of the reaction curves, as assumed by Rand.

Knowing these details, we can also locate the parameter combination at which the zero branch of (8) starts being visited, i.e. some firm starts throwing the competitor out of business. To this end we substitute from (19) into (7) and check when the iterates of these maxima extend to the endpoints of their definition ranges, i.e.

$$
\begin{aligned}
& f\left(\frac{v}{5}\right)=v \\
& g\left(\frac{u}{5}\right)=u
\end{aligned}
$$

Using expressions (7), we can solve for the ratio $v / u$. The conclusion is that the zero branches start being visited only outside the interval:

$$
\frac{5}{41} \leq \frac{v}{u} \leq \frac{41}{5}
$$

i.e. again with a considerable asymmetry in terms of the maximum production capacities.

\section{Examples}

Simulations show that once the Cournot point loses stability, it is replaced by a 4cycle. However, even before, it may coexist with 4-cycles, as we see in Figure 1. The fixed point is represented by the white circle, whereas the 4-cycles, two coexistent to be exact, are shown by the dark circles and squares respectively. The corresponding basins of attraction are indicated by various gray shades.

We can see the cause to this coexistence in Figure 2, where we display the second iterate of the composite function $f \circ g(x)$ for the first variable alone, i.e. $f \circ g \circ f \circ$ $g(x)=(f \circ g)^{2}(x)$. Whenever $f \circ g(x)$ has a 2-cycle, the second iterate has a fixed point, i.e. $(f \circ g)^{2}(x)=x$. For better visibility, we felled the 45 degree line down, 


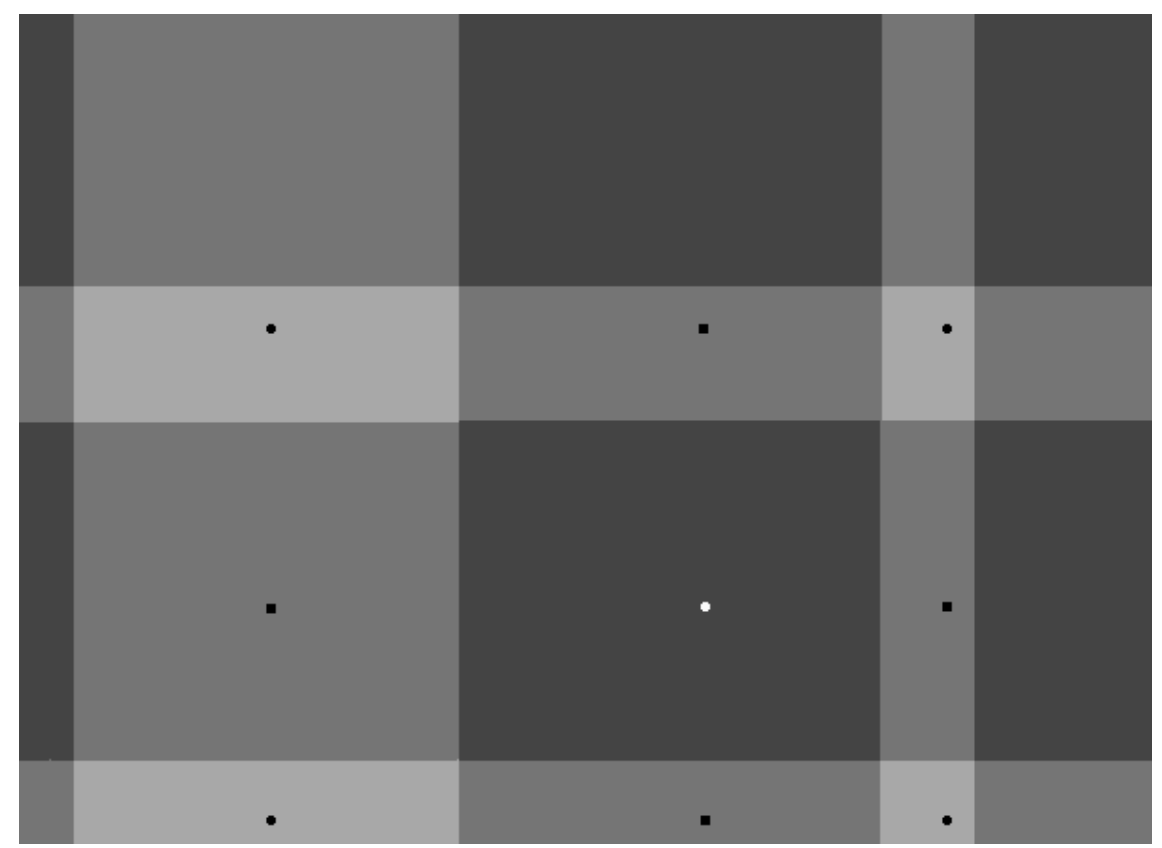

Figure 1: Coexistence of fixed point and two 4-period cycles. Capacity ratio $u / v=7.9$.

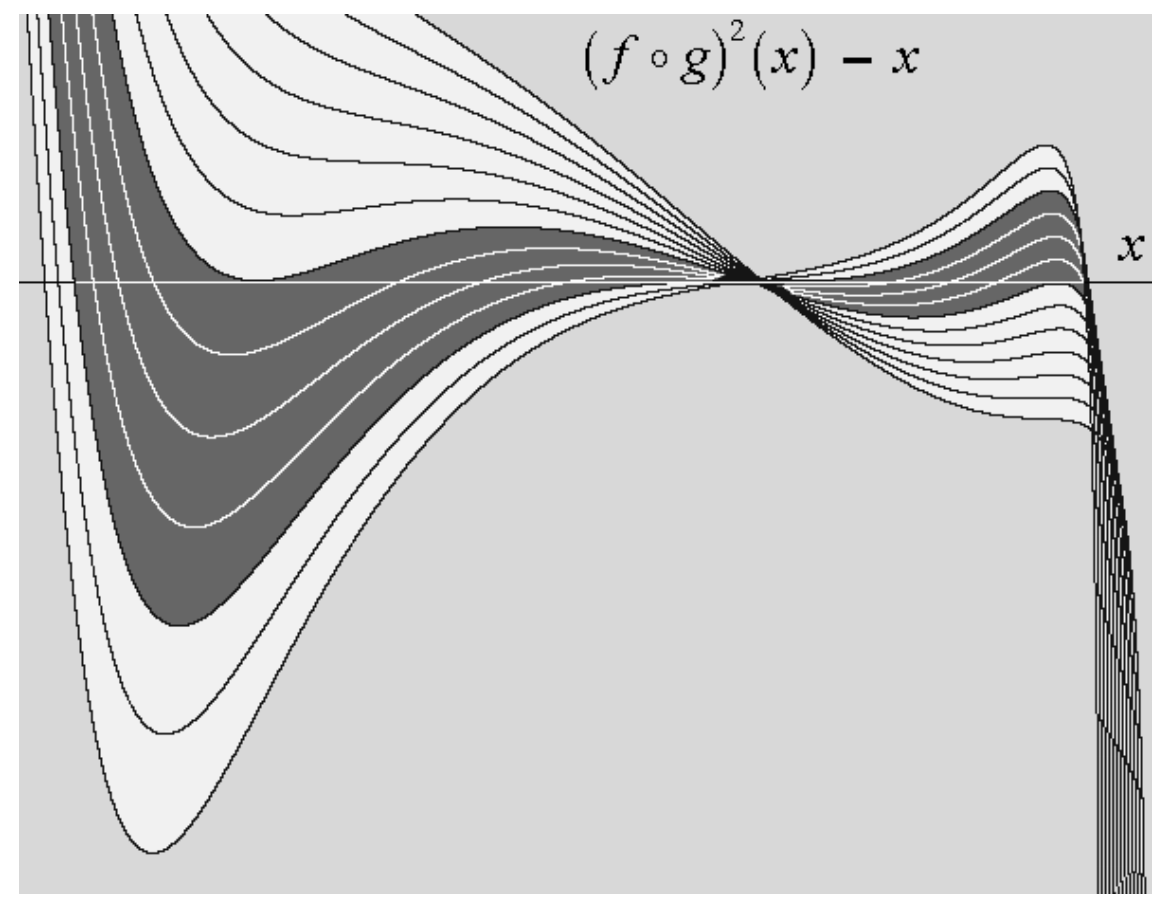

Figure 2: Fixed points and cycles for the composite map $f \circ g$. Curves display the second iterate $(f \circ g)^{2}$ for various capacity ratios $7.7<u / v<8.1$. 


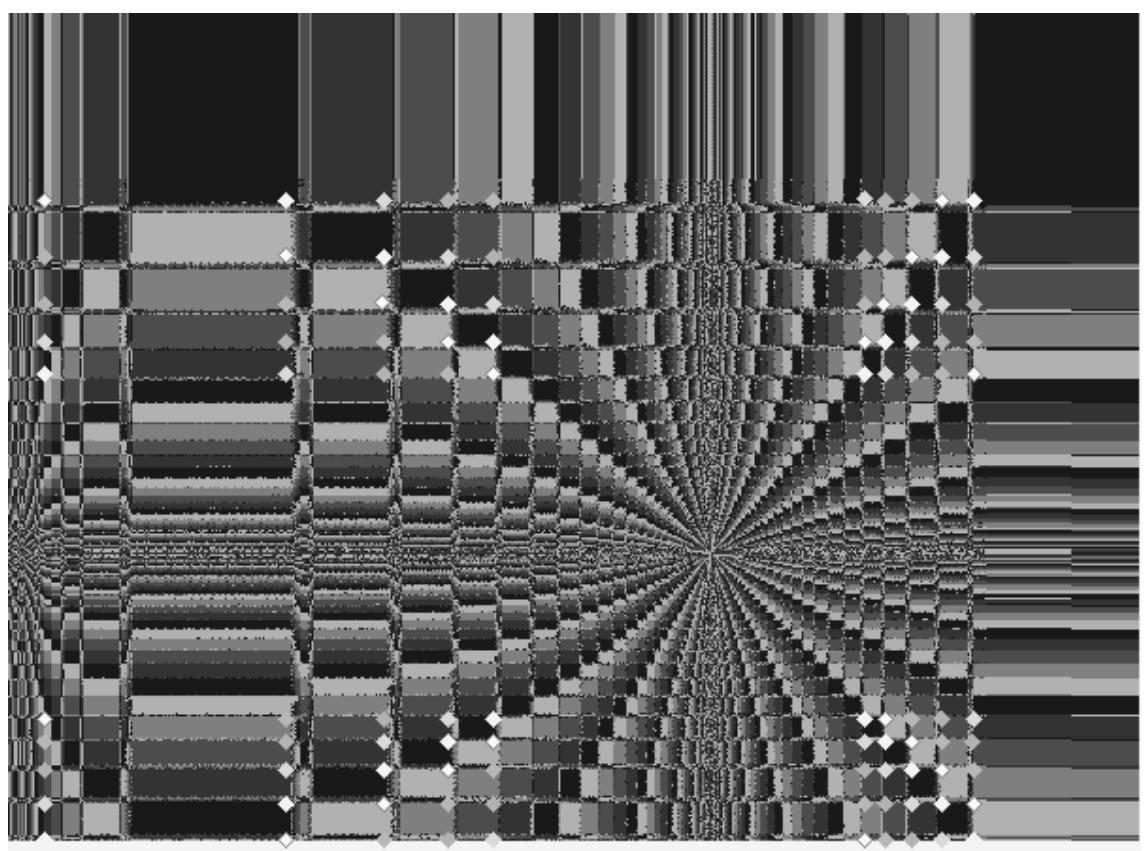

Figure 3: Coexistence of 5 cycles of period 20. Capacity ratio $u / v=8.37$.

i.e. plotted $(f \circ g)^{2}(x)-x$ against $x$. The various curves are drawn for different capacity ratios in the range $7.7<u / v<8.1$, where coexistence of fixed point and cycle occur. As we drew the picture, fixed points are located at the intersections of the respective curve with the horizontal axis (originally the 45 degree line). The curve being second iterate, the intersections can be both 2-cycles and fixed points, stable and unstable. Due to the construction, whenever the curve intersects the horizontal in descending, the point is stable, whenever it intersects in ascending, the point is unstable.

As we see there are three different cases: i) In the upper bright band we have just one intersection, representing the fixed point, which is stable. ii) In the intermediate darker band there are five intersections, again the stable fixed point in the middle, but also the extremal intersections, belonging to a stable 2-cycle. Between these there are two more intersections, belonging to an unstable 2-cycle. iii) Finally, in the lower bright band there are three intersections, the extremal two, belonging to the stable 2-cycle, and the fixed point in the middle, which is now destabilized.

While the parameter ratio changes, the system hence goes from a fixed point, over coexistent fixed point and 2-cycle, to just a 2-cycle. Note that these facts are stated for the composite iteration in $x$ alone. In $x, y$ space these 2-cycles for each variable combine to 4 -cycles in the plane.

Eventually, after the fixed point has lost stability, there follows a period doubling cascade to chaos, until the point where the capacity ratio transgresses the above mentioned critical value 8.2, or its reciprocal, at which (8) replaces the curves by zero segments. After that point there seem to be coexistent cycles, predominantly of period twenty. We illustrate one example of such coexistent cycles in Figure 3.

As we see there is a rectangular pattern of fragmented basins, represented in five different shades. The small tilted squares belong to the attracting cycles. As we see, they are part of a 10 by 10 rectangular lattice. Hence the composite iteration, $f \circ g$ or $g \circ f$, in one variable alone would have 10-period cycles. Quite as predicted by Bischi, Mammana, and Gardini (2000), there are then: 10/2 $=5$ cycles of twice the basic period, i.e., period 20.

Another illustration is provided in Figure 4. In this case we have a 6 by 6 lattice, and there are $6 / 2=3$ cycles twice period 6, i.e. 12-cycles. Despite fewer basins we see that they are much more fragmented. While Figure 3 was taken in the parameter 


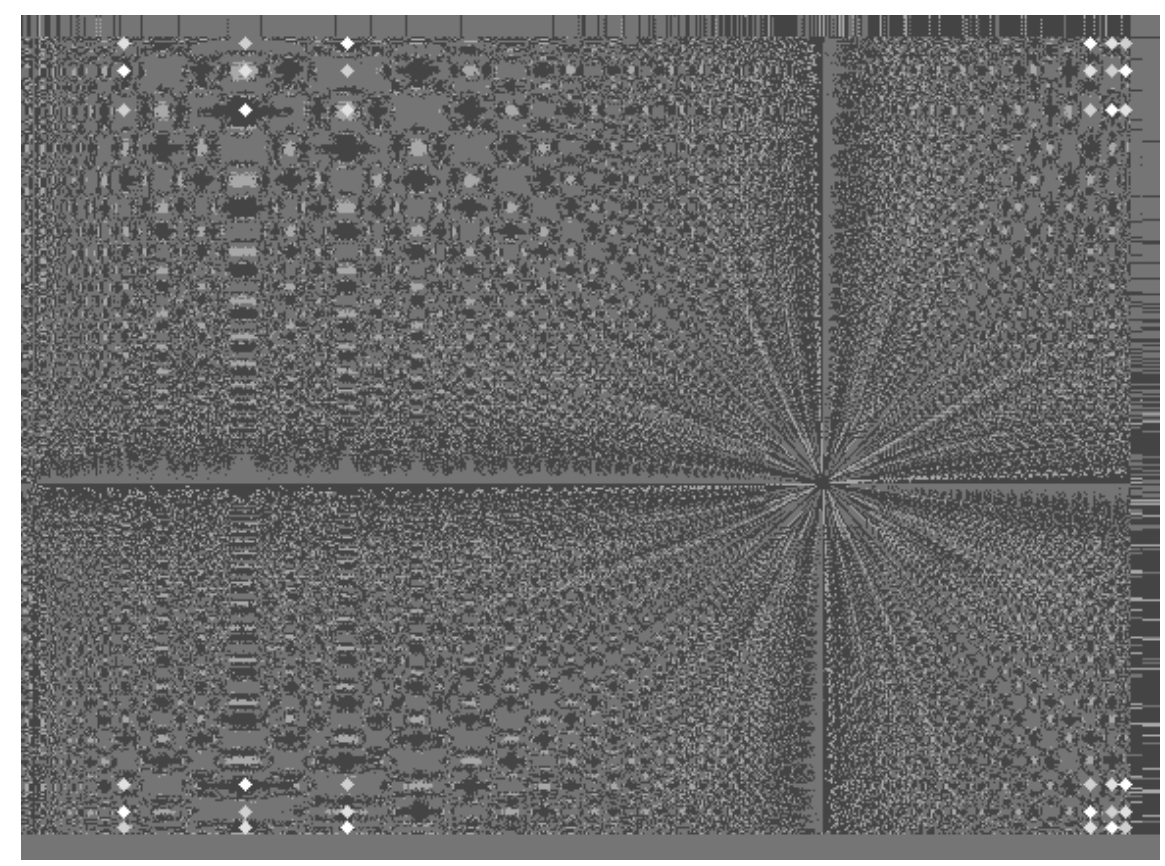

Figure 4: Three coexistent cycles of period 12. Capacity ratio $u / v=8.157$.

range where the zero branches were visited, Figure 4 was taken in the window for 3-period trajectories mid in the chaotic range. (Hence the divisibility by 3). As we can expect, for very slightly different parameter values, the periodic points are replaced by chaotic rectangles, and the fragmented basins merge into a single one. See Figure 5.

\section{Adaptive Adjustment}

We noted that the system discussed until now was essentially a combination of two independent iterations, one for each competitor, no matter whether sequential or simultaneous adjustment was assumed. The iterations, however, become dependent once we introduce adaptive adjustment. The rationale for this is the assumption that producers do not immediately jump to their optimum outputs, given according to their reaction functions from the last moves by the competitors. They just move a bit of the differences from their previous outputs towards these calculated new best replies, thus showing a little conservatism or disbelief in the actual optimality of these best replies.

Through this slight modification, there emerge new types of bifurcation, Neimark instead of flip becoming the basic type of loss of stability for the Cournot point.

To put down the map for the case of adaptive adjustments, write:

$$
\begin{aligned}
x^{\prime} & =\phi(x, y):=(1-\gamma) x+\gamma f(y) \\
y^{\prime} & =\psi(x, y):=\delta g(x)+(1-\delta) y
\end{aligned}
$$

Note that the re-defined functions $\phi(x, y), \psi(x, y)$ now have two arguments each. The new parameters $0 \leq \gamma, \delta \leq 1$ are the adaptation coefficients. When they are unitary, we get the original system back; When they are zero, the competitors show an extreme inertia and never revise a decision once taken.

However, we should again replace the factually senseless negative best replies by 


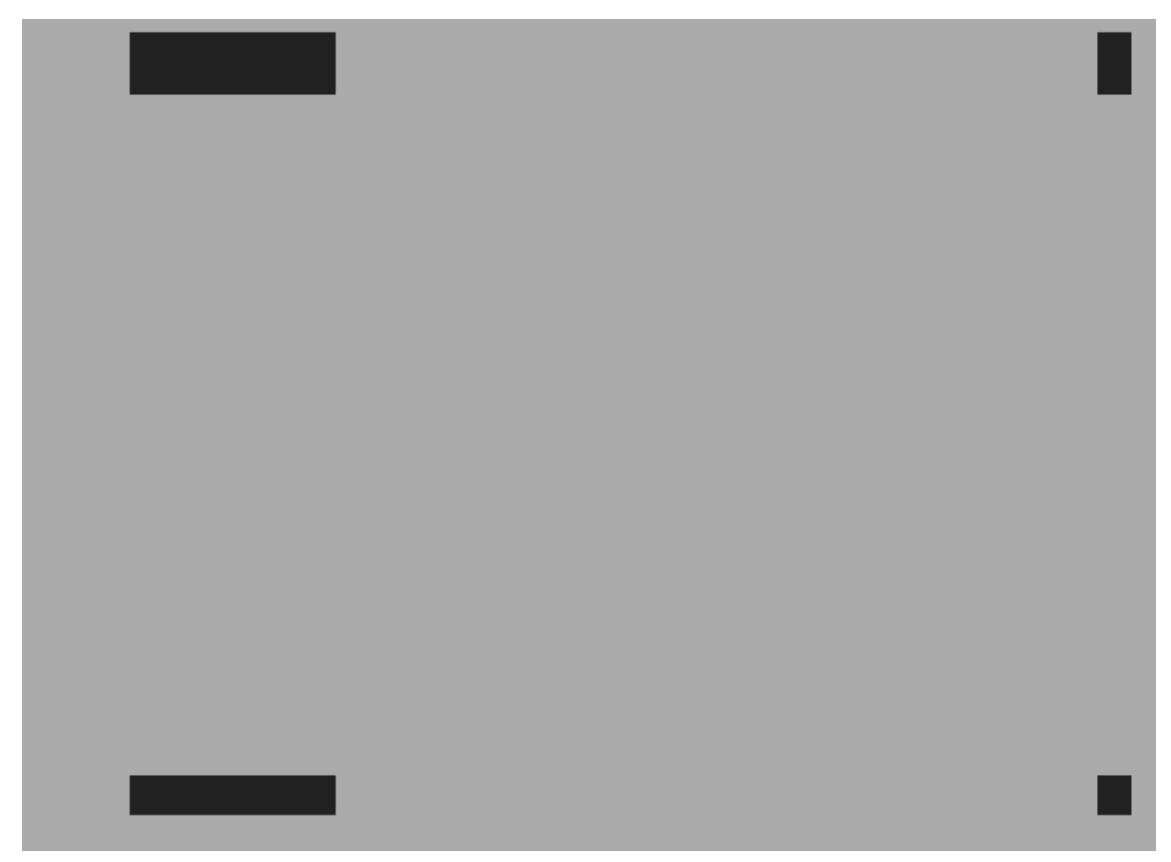

Figure 5: Chaotic rectangles. Capacity ratio $u / v=8.152$.

zeros, and so write:

$$
\begin{aligned}
& x^{\prime}=\Phi(x, y):=\left\{\begin{array}{cc}
(1-\gamma) x+\gamma f(y) & y \leq u \\
0 & y>u
\end{array}\right. \\
& y^{\prime}=\Psi(x, y):=\left\{\begin{array}{cc}
\delta g(x)+(1-\delta) y \leq v \\
0 & x>v
\end{array}\right.
\end{aligned}
$$

It is immediately apparent that the origin and the Cournot equilibrium point, as given by (6) above, are again the only equilibrium points for the adaptive system as well.

\section{Stability in the Adaptive Model}

As for stability of the Cournot point, let us calculate the derivatives from (20), and organize them in the Jacobian matrix for the system.

$$
\left[\begin{array}{cc}
(1-\gamma) & \gamma\left(\frac{2 u+5 y}{2 \sqrt{4 u y+5 y^{2}}}-\frac{3}{2}\right) \\
\delta\left(\frac{2 v+5 x}{2 \sqrt{4 v x+5 x^{2}}}-\frac{3}{2}\right) & (1-\delta)
\end{array}\right]
$$

Substituting the coordinates for the Cournot point simplifies this matrix to:

$$
\left[\begin{array}{cc}
(1-\gamma) & \gamma\left(\frac{u^{2}-v^{2}}{v(2 u+3 v)}\right) \\
\delta\left(\frac{v^{2}-u^{2}}{u(3 u+2 v)}\right) & (1-\delta)
\end{array}\right]
$$

As we see (22) is quite similar to (13). The only difference are the entries in the main diagonal, and the multiplicative coefficients in the off diagonal elements.

The corresponding characteristic equation is easily obtained as

$$
\lambda^{2}-(2-\gamma-\delta) \lambda+(1-\gamma)(1-\delta)+\gamma \delta|J|=0
$$

where $\lambda$ denotes the eigenvalues, or multipliers of the system, and $|J|$ is the determinant of the Jacobian matrix as defined in (14) above. Note from the definition (14) that $|J| \geq 0$. 
Let us now consider different possibilities for the Cournot equilibrium to lose stability. This occurs when a real eigenvalue becomes larger than unity, $\lambda>1$, or less than minus unity, $\lambda<-1$, or when a pair of conjugate complex eigenvalues cross the unit circle in the complex plane. Let us first try the case of real eigenvalues, by substituting $\lambda=1$ in (23). We then find that a number of terms cancel, and obtain $|J|=-1$, which contradicts $|J| \geq 0$.

Next try $\lambda=-1$ in (23). Then $|J|=-(2-\gamma)(2-\delta) /(\gamma \delta)$, which again is negative when $0 \leq \gamma, \delta \leq 1$, and so contradicts $|J| \geq 0$. By conclusion, in the adaptive model, the Cournot equilibrium point cannot lose stability through a fold or flip bifurcation, because no real eigenvalue can take on a unitary absolute value.

What remains is the possibility of a Neimark bifurcation. Suppose we have a pair of conjugate complex eigenvalues

$$
\lambda_{1}, \lambda_{2}=\alpha \pm i \beta
$$

Then the Neimark bifurcation occurs when $\alpha^{2}+\beta^{2}=1$. With these complex eigenvalues, we can also write the characteristic equation as:

$$
\left(\lambda-\lambda_{1}\right)\left(\lambda-\lambda_{2}\right)=\lambda^{2}-2 \alpha \lambda+\alpha^{2}+\beta^{2}=0
$$

Matching constant terms with (23) we find:

$$
(1-\gamma)(1-\delta)+\gamma \delta|J|=\alpha^{2}+\beta^{2}
$$

As $\alpha^{2}+\beta^{2}=1$ at the Neimark bifurcation, we hence have:

$$
|J|=\frac{1}{\gamma}+\frac{1}{\delta}-1
$$

Note that when $\gamma=\delta=1$, then the right hand side becomes unitary, and we get our original bifurcation condition for the nonadaptive model back.

Further, $|J|$ being a quotient with homogeneous fourth order polynomials of $u$ and $v$ in both numerator and denominator, we again find that the bifurcation condition depends on their ratio $v / u$, but not on the variables separately. So, there are in all two parameters in the adaptive model which can cause bifurcations: the compound $1 / \gamma+1 / \delta$ from the two adaptation speeds and the ratio $v / u$ of capacity limits for the firms.

\section{Subcriticality and Coexistence}

Note that what we have investigated is the destabilization of the Cournot equilibrium point through a Neimark bifurcation. These local bifurcations, however, are of two different kinds, supercritical and subcritical. In the first case, an attracting limit cycle is born the moment the fixed point loses stability. In the second case, a pair of stable (outer) and unstable (inner) invariant curves are born, first coincident, then separating with further parameter changes. Upon continued parameter change, separation proceeds until the inner unstable curve collapses around the fixed point, and it is this which finally destabilizes it. Meanwhile there is coexistence of attractors: the fixed point and the stable cycle.

Numerical studies indicate that the loss of stability is in fact due to a subcritical Neimark bifurcation. Accordingly, complex outcomes of the model start with the birth of the pair of invariant curves already, and not with the destabilization of the fixed point. This first gloval bifurcation, however, is difficult to establish analytically for the adaptive model, so we have to rely on numerical explorations. In the cases of coexistence it will be of interest to draw the basins of coexistent attractors.

The practical significance of subcriticality in terms of economics is connected with the contollability of a dynamical process. When a fixed point has lost stability through a supercritical bifurcation, the process can always be stabilized at the fixed 


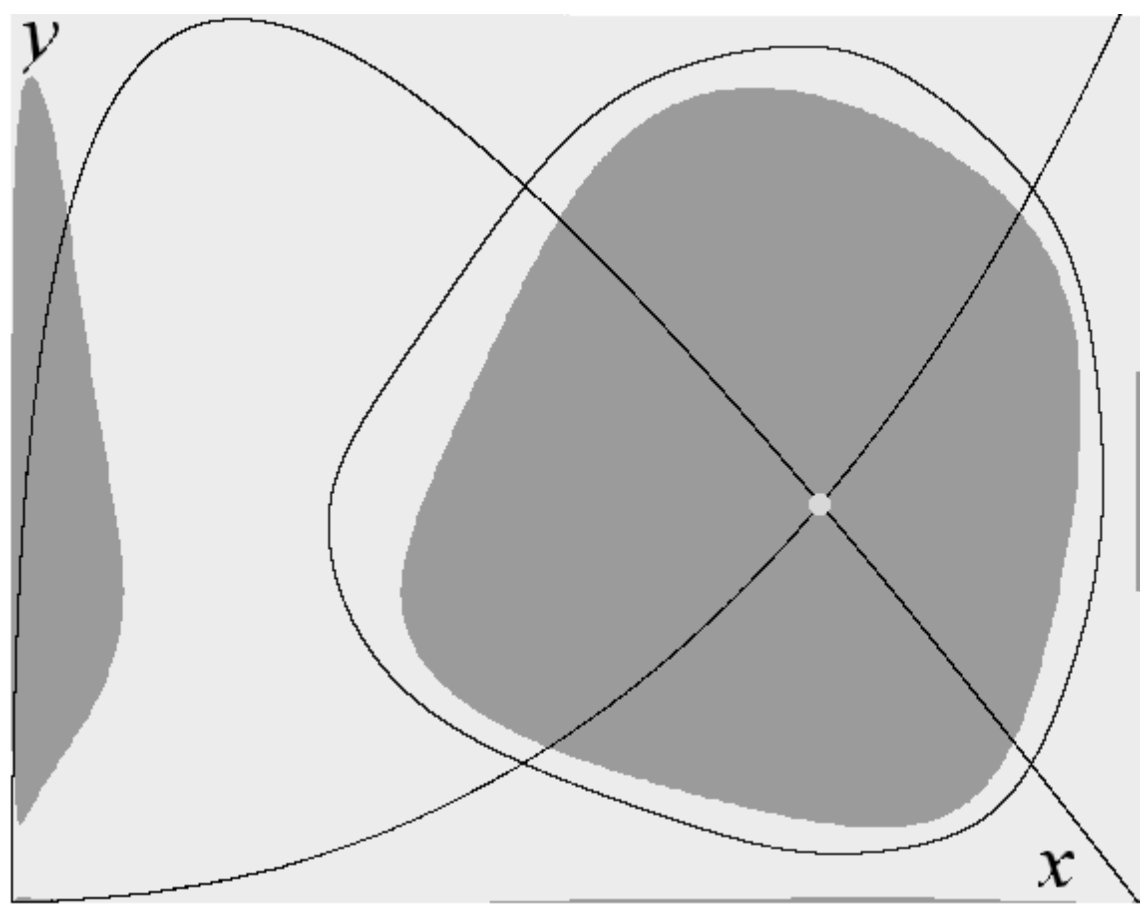

Figure 6: Coexistence of fixed point and invariant curve. Capacity ratio $u / v=7.975$. Adaptation coefficients $\gamma=\delta=0.9925$.

point through a small adjustment of parameters or initial conditions. This is not possible when the bifurcation is subcritical, because the process then makes an immediate leap from the fixed point, and so cannot be brought back to it by marginal moves.

Figure 6 displays a case of coexistence for fixed point and closed invariant curve. The dark area still attracts to the fixed Cournot point, whereas initial conditions in the bright area end up at the invariant curve. Note that in addition to the immediate attraction basin for the fixed point, encircled by the unstable invariant curve, there exist preimages of this immediate basin.

Coexistence can also occur for more complex attractors, such as periodic of different periodicities, or chaotic.

\section{Periodic Solutions and Other}

Let us now note some basic facts connected with the loss of stability for the Cournot equilibrium point. These matters are much simpler for supercritical bifurcations. Then there exists a theorem stating that, if there is no so called strong resonance, then the fixed point always gives way to a closed invariant curve. As we do not deal with a continuous time system, this invariant curve would not be traced in continuous motion, like a limit cycle after a Hopf bifurcation. Rather, it would be traced in showers of points which precipitate at various locations on the curve, and only after a while it gives the impression of a dense curve, i.e. provided we deal with an irrational rotation number. Though dense, it is, however, not continuous, as there are infinitely many gaps at all the rational points.

If the rotation number is rational, then there seems to be no closed curve. In simulation one sees just a finite collection of points. The trajectory is periodic. However, the invariant curve still exists. Between the attracting points, which one sees at simulation, there are equally many saddle points, which cannot be seen as they are unstable. It is the unstable manifolds of these saddle points, heading for the stable periodic points, that form the invariant curve. 
The aforementioned theorem states that, when there are no strong resonances, then at the moment of bifurcation we do not get any periodic orbit, but a quasiperiodic one, which is dense on the invariant curve. The proof of the theorem belongs to advanced mathematics. For references see Kuznetsov (1995). Strong resonance is defined as resonance with frequencies 1:1, 1:2, 1:3, or 1:4.

The complex eigenvalues of the characteristic equation (23) at the moment of bifurcation can be written:

$$
\begin{aligned}
& \lambda_{1}=\alpha+i \beta=\cos \theta+i \sin \theta \\
& \lambda_{2}=\alpha-i \beta=\cos \theta-i \sin \theta
\end{aligned}
$$

because we then have $\alpha^{2}+\beta^{2}=1$. In case of a rational rotation number:

$$
\theta=\frac{2 m \pi}{n}
$$

where $m, n$ are positive integers with no common divisor.

For strong resonance one has $n \leq 4$. The number $m$ has no importance for this issue. It is $n$ which represents the number of points in a periodic solution, $m$ is just the number of rounds along the invariant curve needed to trace all these points.

We can now check out the real part of the eigenvalues:

$$
\alpha=\operatorname{Re} \lambda_{1,2}=\cos \left(\frac{2 m \pi}{n}\right)
$$

From the characteristic equation (23), we immediately see that it equals minus half the coefficient of the linear term, i.e.

$$
\alpha=1-\frac{\gamma+\delta}{2}
$$

Collecting results:

$$
\cos \left(\frac{2 m \pi}{n}\right)=1-\frac{\gamma+\delta}{2}
$$

Substituting different strong resonances $n=2,3$, 4 , we see that for $n=2$, we must have $\gamma+\delta=4$, for $n=3, \gamma+\delta=3$, and for $n=4, \gamma+\delta=2$. As $0 \leq \gamma, \delta \leq 1$, only the last case is possible, and then only when $\gamma=\delta=1$, i.e. when we are back to the non-adaptive model. Finally, for $n=1$ we must have $\gamma+\delta=0$, which means $\gamma=\delta=0$. Hence, in the adaptive model, the condition for no strong resonance is fulfilled, and we could expect bifurcation to a closed invariant curve. At least this would be so if we dealt with a supercritical bifurcation.

Unfortunately, things are not that simple, as the bifurcation is subcritical. The bifurcation moment signifies that an unstable invariant curve collapses around the fixed point and so destabilizes it, as described above. However, the unstable invariant curve was born together with an outer stable one, and the moment the Cournot point is destabilzed through a Neimark bifurcation, this stable invariant curve may be located at considerable distance from the Cournot point, and may already have undergone bifurcations to periodicity or chaos. This indeed is indicated by some of the results of numerical experiment.

We can also use (25) to find out at which points of the curve (24) in $\gamma, \delta$ parameter space, loss of stability occurs with any given rational rotation number. Say for instance that we want to check $n=6$. Substituting into (25), we find $\gamma+\delta=1$, so, along with $|J|=1 / \gamma+1 / \delta-1$, we can for any given $|J|$ pick the points in which 6-periodic cycles bifurcate from the fixed Cournot point. The regions in parameter space for given periodicities, which bifurcate out from the curve for Neimark bifurcation. are called Arnol'd tongues. For a supercritical bifurcation they set out from all the rational points of the bifurcation curve itself.

Everybody knows that the rational numbers, being a countable set, are outnumbered by the irrationals, so the probability of a periodic cycle right at the moment of bifurcation is zero. However, once the exact bifurcation curve is left, 


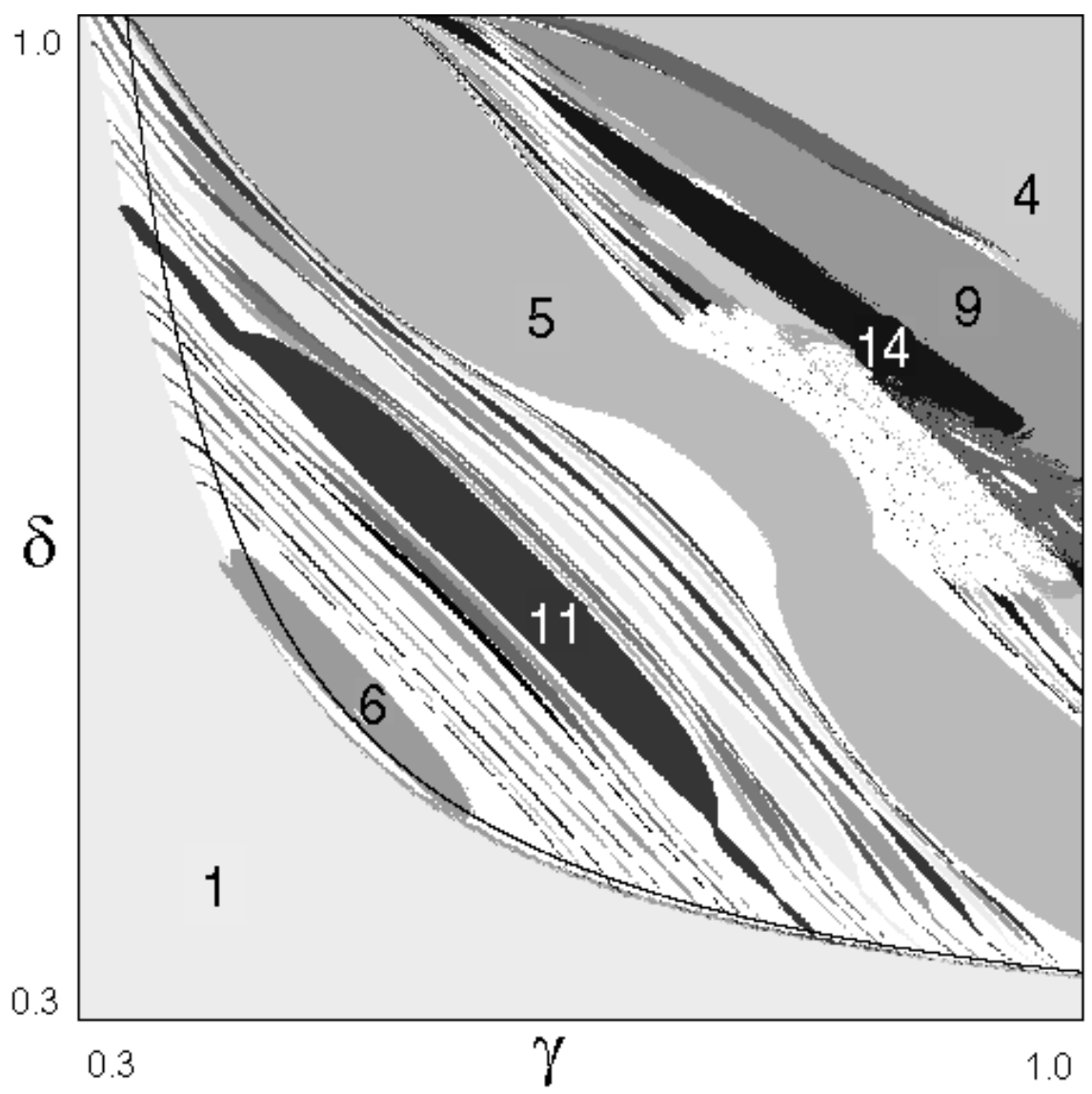

Figure 7: Bifurcation diagram with Arnol'd tongues in parameter $\gamma, \delta$-space. Capacity ratio $\mathrm{v} / \mathrm{u}=20.0857$

things change. The Arnol'd tongues soon grow fat, and, being infinite in number they cover a major part of space. So, even if supercritical bifurcations start out with closed invariant curves, they soon bifurcate again in periodic trajectories. As the tongues may overlap, there may arise coexistence of attractors.

The periodic tongues can be seen in Figure 7 , which displays the bifurcation diagram in $\gamma, \delta$-parameter space. Actually we have three parameters: $\gamma, \delta$, and $|J|$, or rather the capacity ratio $v / u$. As we recall, $|J|$ depends on this capacity ratio $v / u$ alone. In order to be able to draw a plane picture, let us fix $|J|=3$. A capacity ratio of approximately 1:20 will do. Then the Neimark bifurcation curve becomes:

$$
\frac{1}{\gamma}+\frac{1}{\delta}=4
$$

It can be seen as the hyperbola shaped curve through the square $0.3 \leq \gamma, \delta \leq 1$ displayed in the picture. Below it the fixed Cournot point is stable.

However, it is time to again recall the additional complications due to subcriticality. Even though we can still find the locations of different rational bifurcations from the Neimark bifurcation curve, the tongues do not really set out from that curve, they are already thick when they cross it, and below it the periodic attractors may coexist with the stable fixed point. As we see in Figure 7, the periodic tongues actually start below the bifurcation line and protrude through it.

What really happens the moment the tongues arise is mathematically not known and seems to provide an important area for further research.

We already found that a 6-period cycle bifurcates for $\gamma+\delta=1$. This curve is actually tangent to $1 / \gamma+1 / \delta=4$, in its midpoint where $\gamma=\delta=1 / 2$. So, we conclude that the 6-cycle bifurcates from the midpoint of the bifurcation curve. This also is exactly what we can see in Figure 7, and we also find 6-cycles when we 


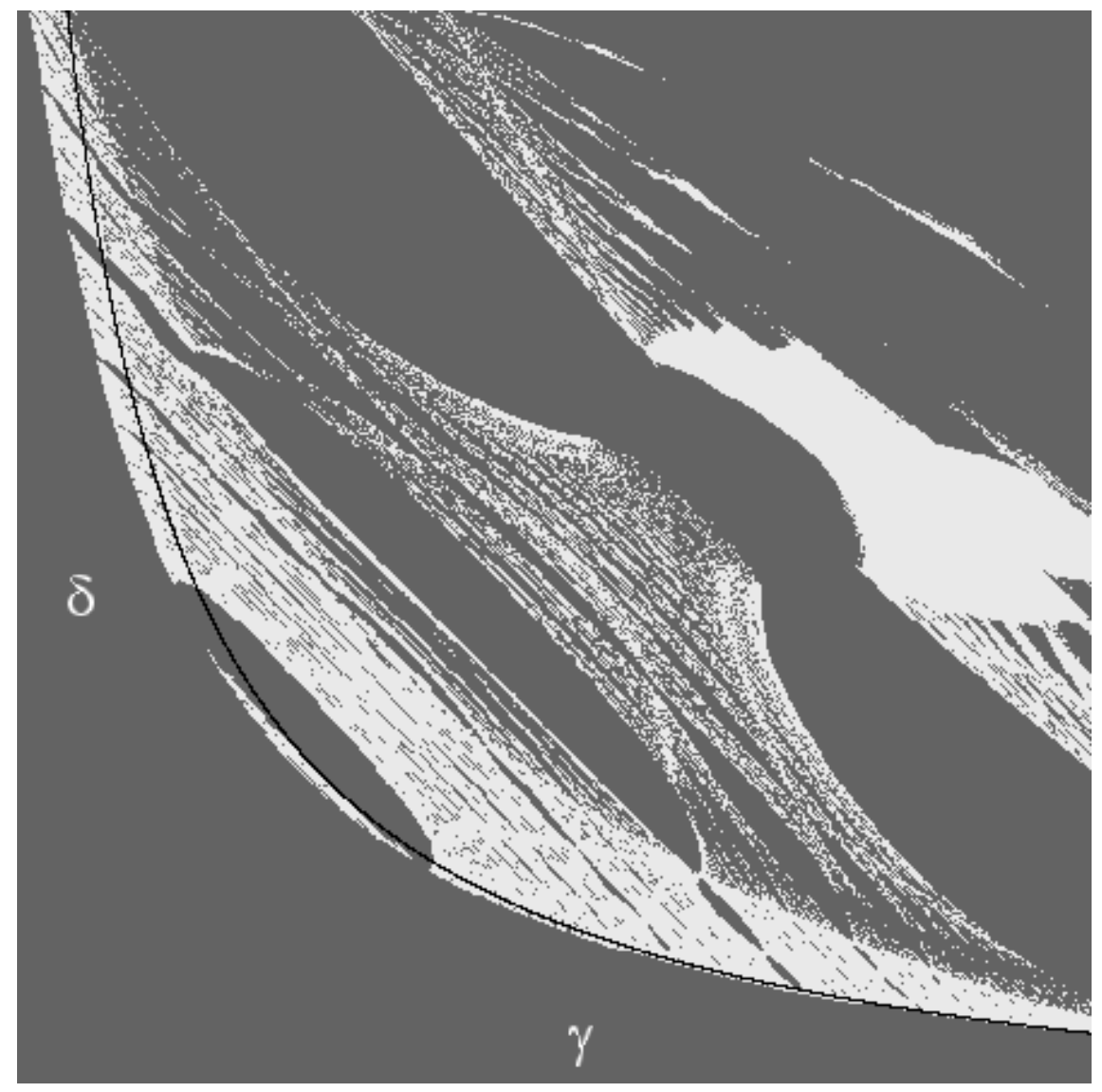

Figure 8: Parameter $\gamma, \delta$-space with bright area representing cases with positive Lyapunov exponent. Capacity ratio v/u=20.0857. Parameter ranges $0.3 \leq \gamma, \delta \leq 1.0$

simulate the system for adjustment speeds both around one half, and a capacity ratio around one to twenty.

The different Arnol'd tongues have different shades. As we traverse the bifurcation diagram along the main diagonal, the system passes through regions of 6-, 11-, 5-, 14-, 9-, and 4-period cycles. Most likely there are coexistent cycles of different periods within these tongues, but we cannot see such overlaps, as we chose shade according the lowest period.

We only checked periods up to 45 . The white area in-between can correspond to: either cycles of higher periods, or quasiperiodic orbits, or else chaotic motion. Bifurcation diagrams are useful when we want to detect interesting parameter regions for bifurcation scenarios. In Figure 8 we give some complementary information, where the areas with positive Lyapunov exponent are indicated in white. Note how Figs. Figure 7 and Figure 8 fit together like hand in glove. We conclude that the trajectories are predominantly chaotic outside the periodic tongues. Also note that the chaotic attractors exist even below the bifurcation curve.

Recall that we fixed one parameter. To get full information we should stack such bifurcation diagrams, using the third parameter, on top of each other so that we get a picture in three dimensions. It is, however, always difficult to visualize three dimensional pictures, especially because the structure may be similar to the peels of an onion, where the outer peels hide the inner structure.

We obtain complementary information from another plane section of the three dimensional $\gamma, \delta, v / u$-space, by putting $\gamma=\delta$ on the horizontal, and $v / u$ on the vertical axis. Such a bifurcation diagram is shown in Figure 9. In three dimensional parameter space Fig. Figure 9 is perpendicular to Figure 7 , and intersects the latter along the diagonal (where $\gamma=\delta$ ). In the new bifurcation diagram the Arnol'd 


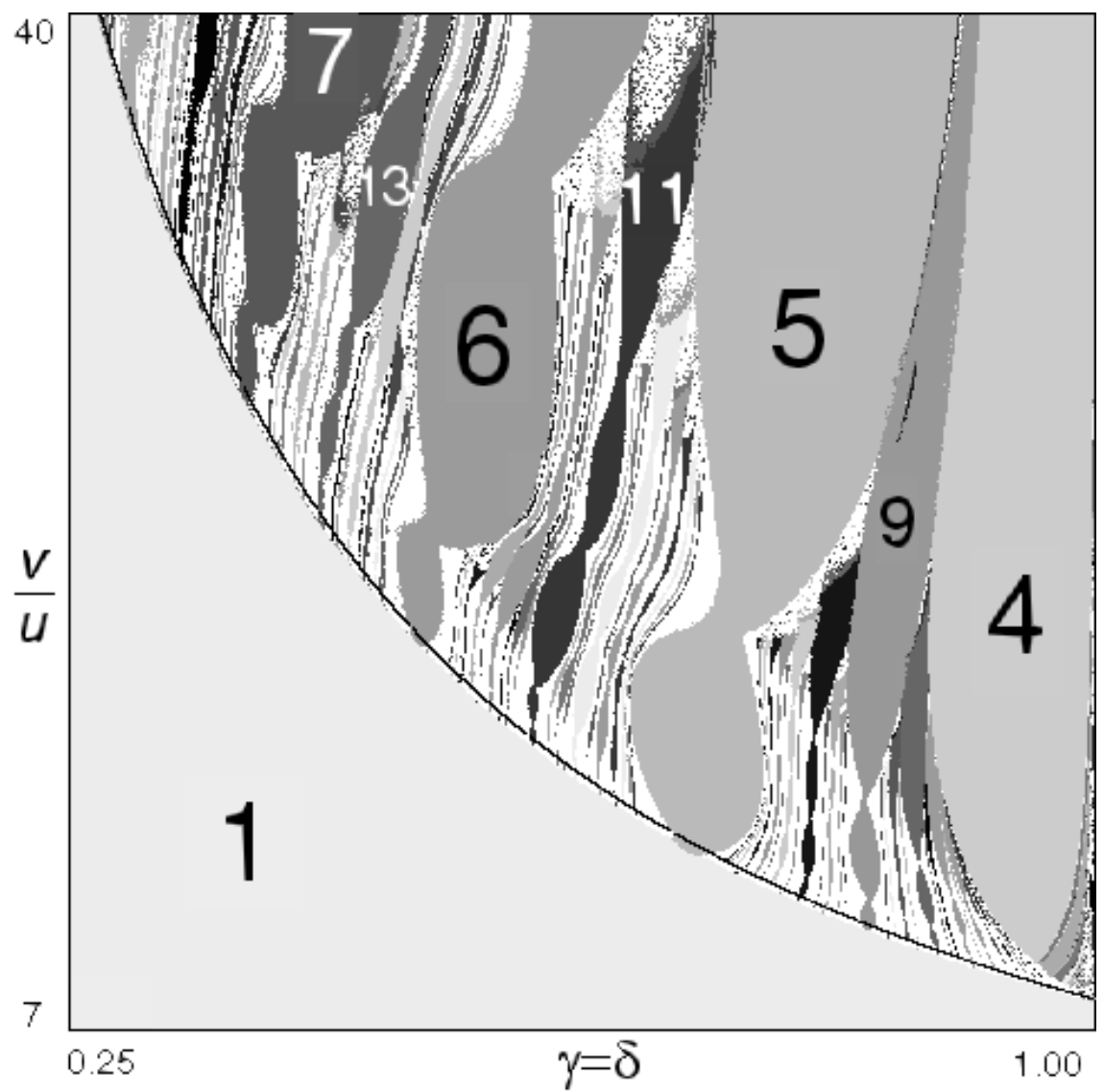

Figure 9: Bifurcation diagram with equal adaptation speeds $\gamma=\delta$ on the horizontal and the capacity ratio $v / u$ on the vertical axis. 


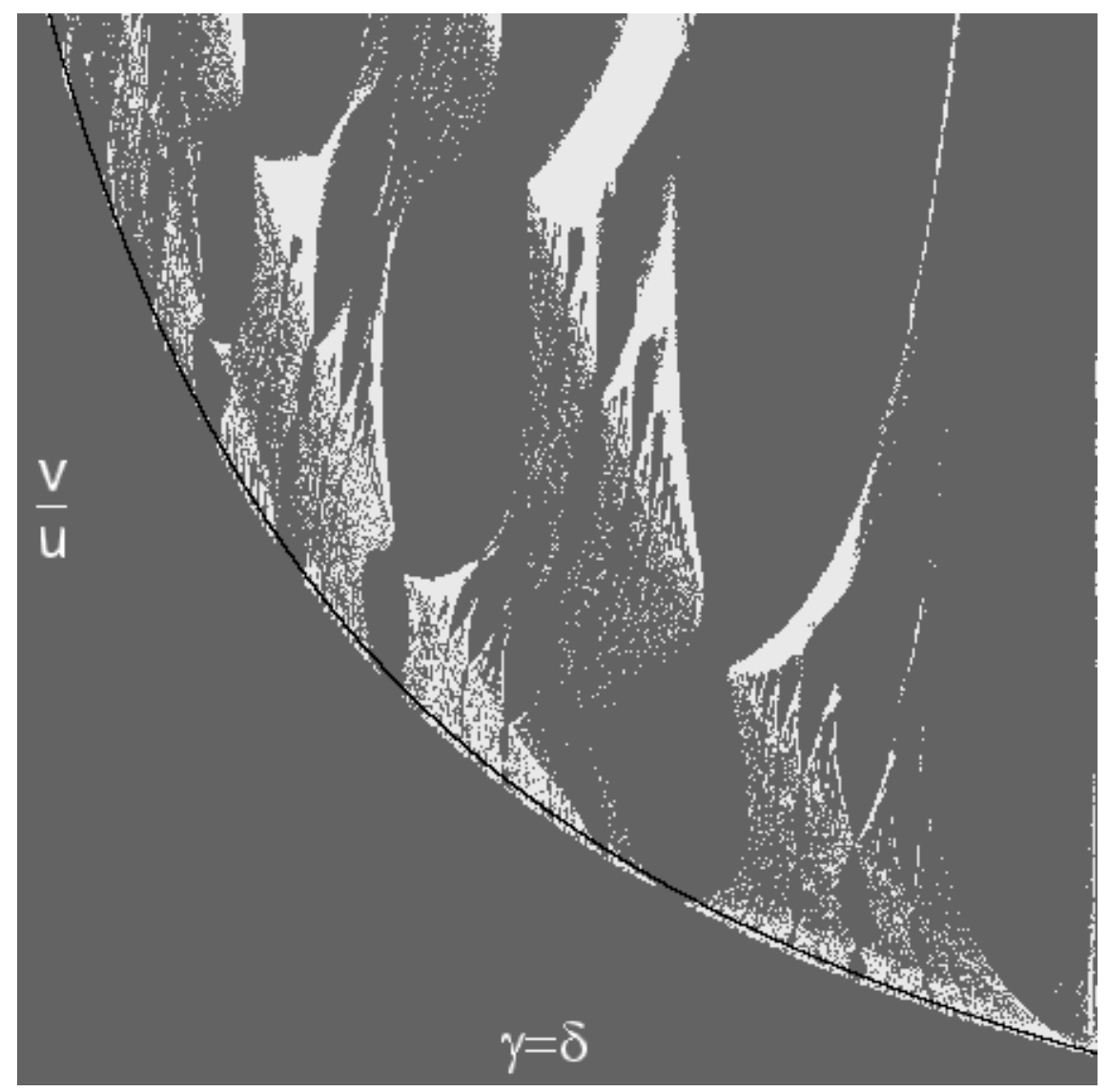

Figure 10: Area of positive Lyapunov exponent (bright) with equal adaptation speeds $\gamma=\delta$ on the horizontal and the capacity ratio $v / u$ on the vertical axis. Parameter ranges $0.25 \leq$ $\gamma, \delta \leq 1.00,7 \leq v / u \leq 40$.

tongues take a more normal shape, though again they start below the bifurcation curve, and cross it.

The bifurcation points can be calculated, using (25). We see in order from right to left the most prominent tongues of resonance 1:4 at $\gamma=\delta=1-\cos (2 \pi / 4)=1$, next 2:9 at $\gamma=\delta=1-\cos (4 \pi / 9) \approx 0.826,1: 5$ at $\gamma=\delta=1-\cos (2 \pi / 5)=$ $(5-\sqrt{5}) / 4 \approx 0.6910,2: 11$ at $\gamma=\delta=1-\cos (4 \pi / 11) \approx 0.584$, then $\gamma=$ $\delta=1-\cos (2 \pi / 6)=0.5$, as we already know, further 2:13 at $\gamma=\delta=1-$ $\cos (4 \pi / 13) \approx 0.432$, and finally $1: 7$ at $\gamma=\delta=1-\cos (2 \pi / 7) \approx 0.376$. The thin tongues without numbers in the upper left corner of the picture correspond to $2: 15$ and 1:8. Further, to the right, between the big 1:4 and 2:9 there is a visible 3:13 tongue, and between 2:9 and 1:5 a 3:14 tongue.

Once the $\gamma=\delta$-parameters are known, we can use the Neimark bifurcation condition (24) together with the definition (14) of $|J|$ to solve for the bifurcation values of $v / u$. Keeping $\gamma=\delta$ fixed, and changing $v / u$ slowly from the bifurcation value, we are likely to see interesting bifurcation sequences. Especially for resonance with numerators larger than unity, we find that the tongues stop abruptly and give way to other periodicities, before yielding to chaos. The 2:11 tongue is one instance, as is the 3:14. We also see likely overlaps where we might find coexistent attractors.

Like we gave the picture for positive Lyapunov exponents as complementary information for the previous parameter plane, we display Figure 10 as a companion to Figure 9. Again we see how the pictures fit together.

In Figure 11 and Figure 12 we illustrate two cases of coexistent periodic attractors and their basins. In Figure 11 there are one 7-cycle (the same as 4:28) and one 27cycle (from the 4:27 tongue). Likewise in Figure 12 there are one 6-cycle (same as 5:30) and one 29-cycle (from the 5:29 tongue). These are but two examples of 


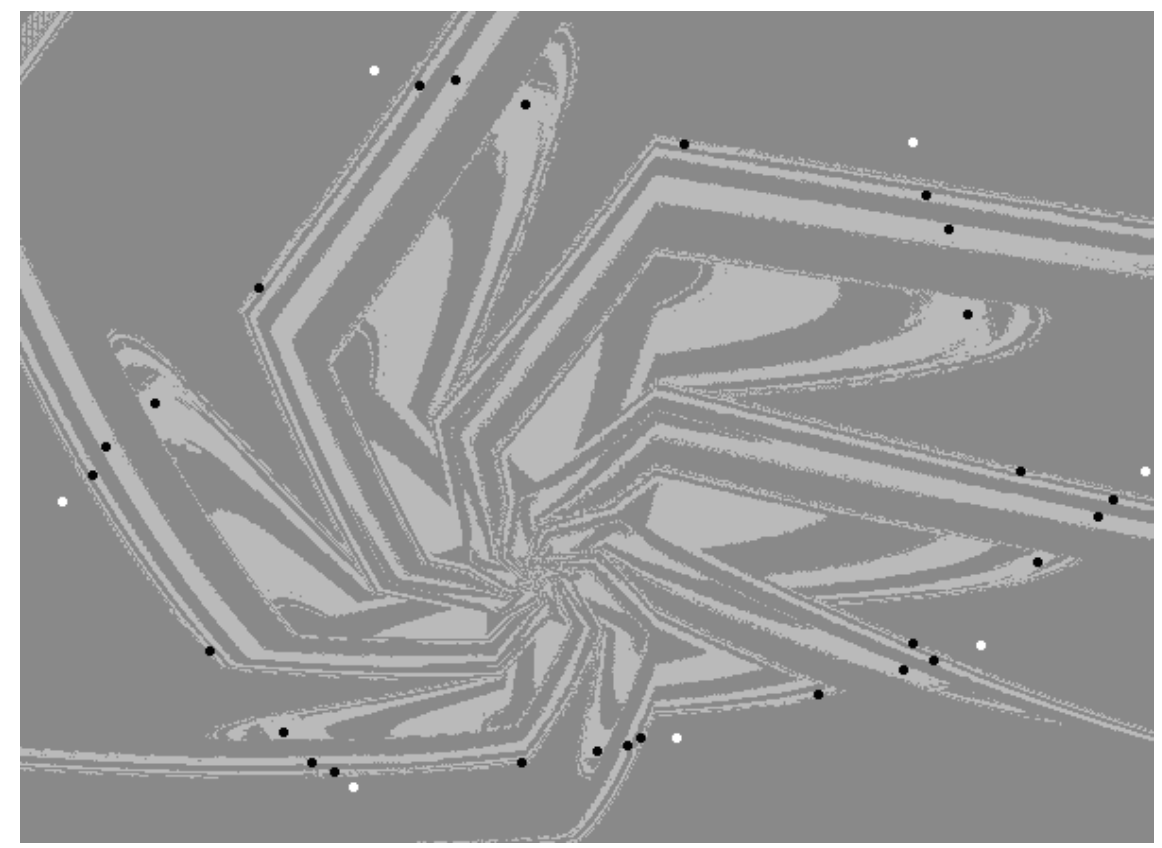

Figure 11: Coexistence of 7-cycle and 27-cycle at $\gamma=\delta=0.47$ and $v / u=38$. Picture area $0.08 \leq x \leq 0.172$ and $0 \leq y \leq 0.01$

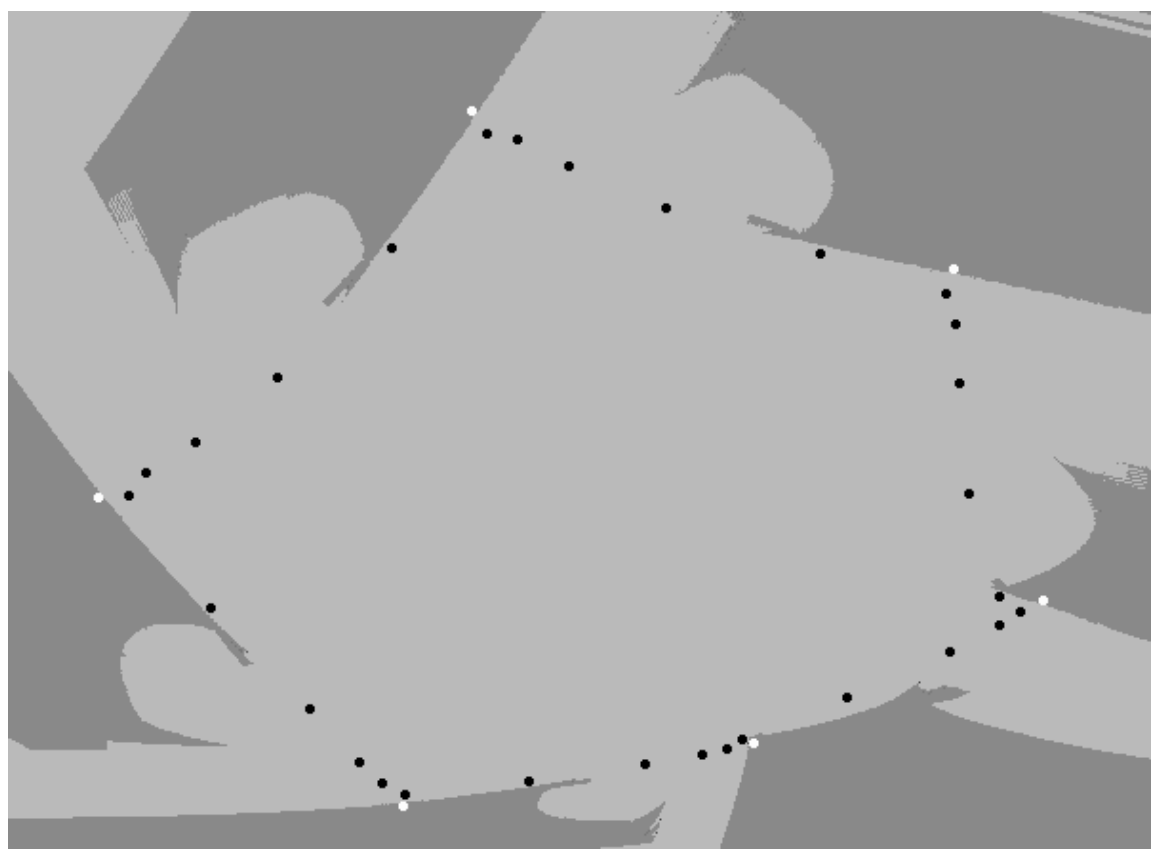

Figure 12: Coexistence of 6-cycle and 29-cycle at $\gamma=\delta=0.575$ and $v / u=23$.4. Picture area $0.1 \leq x \leq 0.275$ and $0 \leq y \leq 0.025$ 


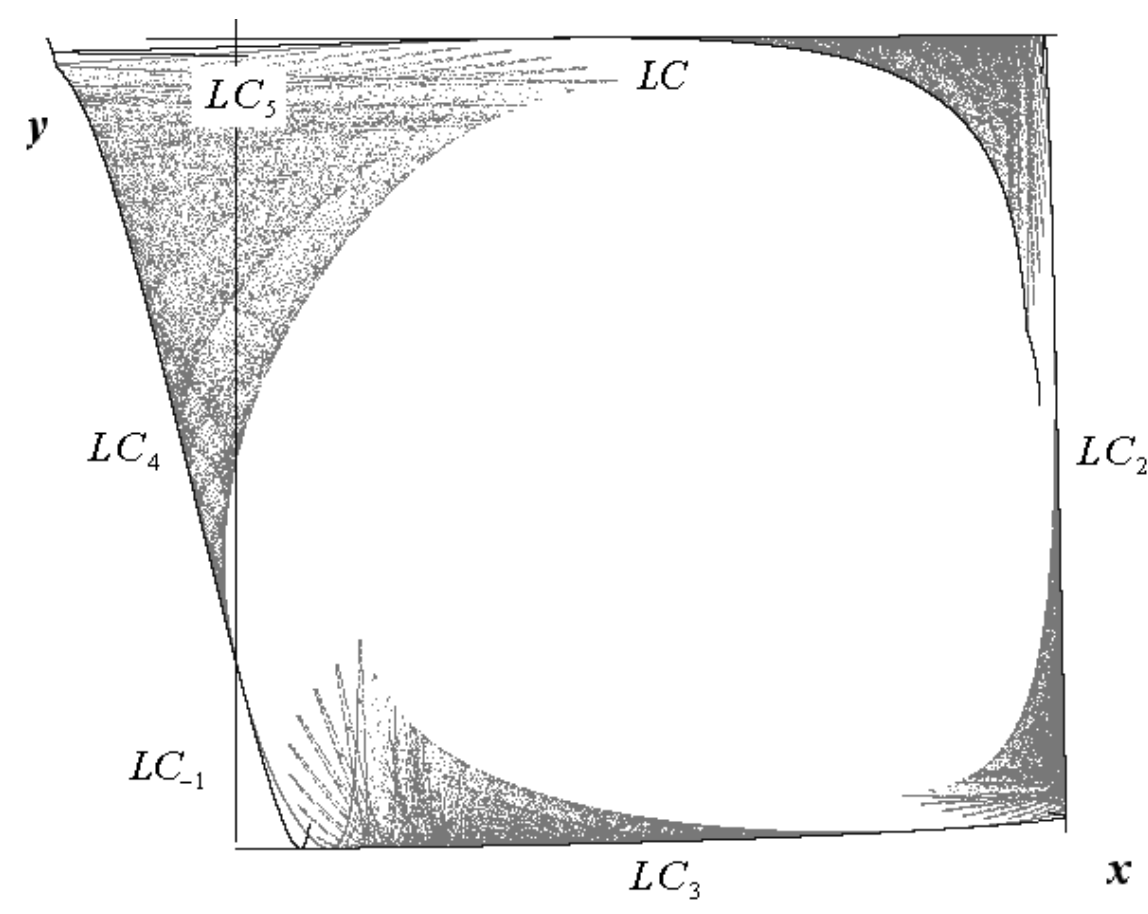

Figure 13: Absorbing area delimited by 5 critical lines. Capacity ratio $\mathrm{v} / \mathrm{u}=8.21$. Adaptation coefficients $\gamma=0.975, \delta=0.995$. Lyapunov exponent $\approx 0.055$

coexistence which we can find by exploring the bifurcation diagrams.

\section{Critical Lines and Absorbing Areas}

Like most other maps, (20) is non-invertible. This means that upon iteration it folds the plane, so that some points have multiple preimages, i.e. different points map into one and the same point. This, of course, complicates some things, but it also simplifies other. In particular, it is possible to find attractor shapes in terms of the absorbing areas, bounded by so called critical lines. These results are due to early work by Mira and Gumowski, which only recently have come in more widespread use. See for instance Abraham, Gardini, and Mira (1997).

The critical lines are defined as follows. The first one, usually denoted $L C_{-1}$, is obtained by putting the Jacobian determinant of the iterated map equal to zero. Observe that we are now not talking about the Jacobian in the Cournot point $|J|$, but of the general Jacobian (21). Further, we put its determinant equal to zero, not to unity as before, because we are not checking stability, but where the plane is folded. Hence:

$$
\left|\begin{array}{cc}
(1-\gamma) & \gamma\left(\frac{2 u+5 y}{2 \sqrt{4 u y+5 y^{2}}}-\frac{3}{2}\right) \\
\delta\left(\frac{2 v+5 x}{2 \sqrt{4 v x+5 x^{2}}}-\frac{3}{2}\right) & (1-\delta)
\end{array}\right|=0
$$

defines the locus of $L C_{-1}$ which is a curve in $x, y$-space. Written out we get the condition:

$$
\frac{(1-\gamma)(1-\delta)}{\gamma \delta}=\left(\frac{2 u+5 y}{2 \sqrt{4 u y+5 y^{2}}}-\frac{3}{2}\right)\left(\frac{2 v+5 x}{2 \sqrt{4 v x+5 x^{2}}}-\frac{3}{2}\right)
$$

This is the equation of a pair of hyperbola like curves. In the following example $\gamma, \delta$ are close to unity, which makes the left hand side of (26) very small. Given this, the hyperbolas have very sharp corners, they are almost a cross of straight 


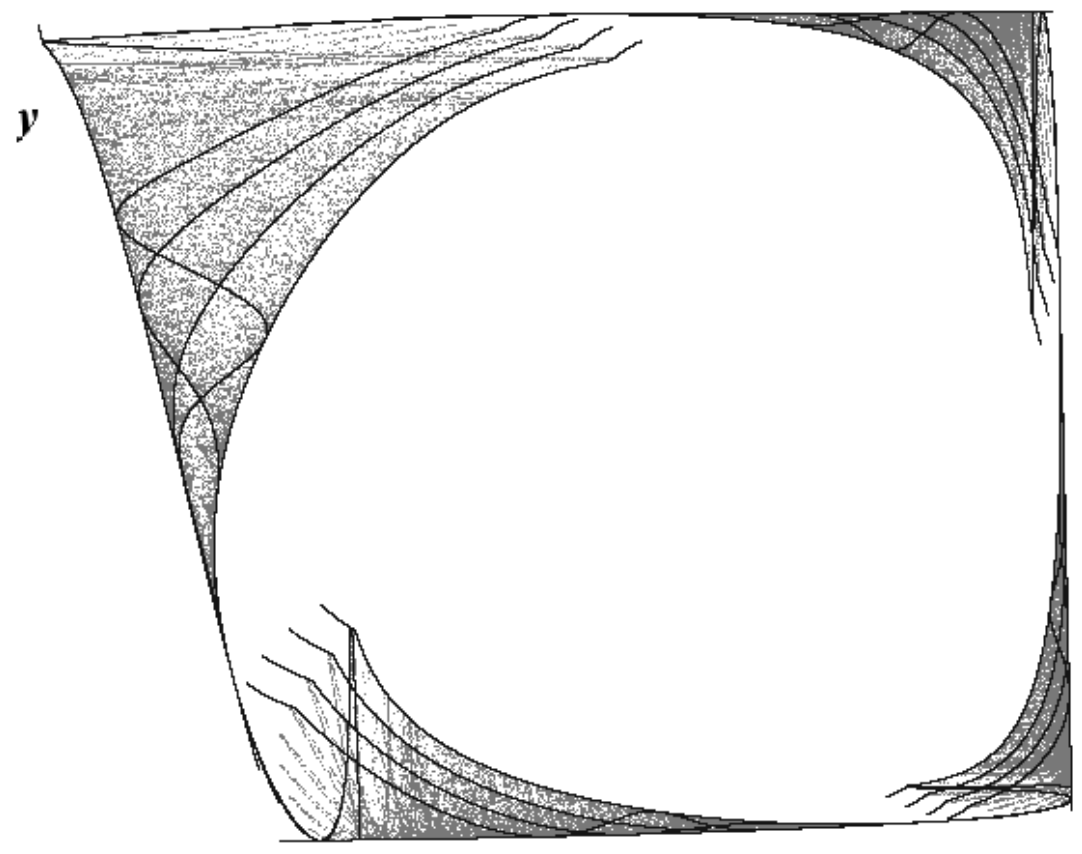

$\boldsymbol{x}$

Figure 14: Absorbing area delimited by 20 critical lines. Capacity ratio $\mathrm{v} / \mathrm{u}=8.21$. Adaptation coefficients $\gamma=0.975, \delta=0.995$.

lines, obtained by putting each of the factors in the right hand side of (26) equal to zero As we have seen, these are the derivatives of the reaction functions, so they become zero when the reaction functions have their maxima. We also know that the factors become zero when $y=u / 5$ and $x=v / 5$ respectively, which we can check again in (26). The piece of $L C_{-1}$ which we need to iterate is in fact to a very good approximation a vertical straight line segment $x=v / 5$, seen to the left in Figure 13 .

Upon iteration of this line through the map (20), we obtain: first $L C$, then $L C_{2}$, $L C_{3}, L C_{4}$ and $L C_{5}$, displayed clockwise in the picture, the last closing the final gap of the absorbing area boundary.

We note two important things:

i) The train of critical lines meet at tangency, so, as the critical line and its forward iterates fold the plane, they represent lines beyond which no iterations can go. The area enclosed is trapping, and called absorbing area. It also is contained in a neighborhood from which iterations enter this trapping area in a finite number of steps.

ii) The critical lines displayed contain a manifestly chaotic looking attractor, obtained through simulating the map from an arbitrary point in its attraction basin. Note that the critical lines are tightly wrapped around the gray attractor. Accordingly, their shape gives an accurate outline of the attractor itself, even if we do not care to simulate the process. With just four iterates we get the outer boundary. Adding critical lines of higher rank, we can also delineate the hole inside the attractor, and inner structures such as densifications of points. See Figure 14.

Besides giving information about shapes of attractors, the critical lines are important tools for understanding various bifurcations, due to intersections of critical lines with invariant curves, or with the boundary of the attraction basin.

\section{Conclusion}

By conclusion we note that the duopoly model with capacity constraints is able to produce very complex dynamic behaviour in terms of basin fagmentation, and in 
terms of the variety of attractors, periodic, quasiperiodic, and chaotic. To address the basic question about whether an increased number of competitors is bound to stabilize the system and eventually lead to competitive equilibrium, we would need further studies about oligopoly involving more competitors than two.

\section{References}

X Abraham, R., Gardini, L., and Mira, C., 1997, Chaos in Discrete Dynamical Systems: A Visual Introduction in 2 Dimensions, Springer-Verlag

$X$ Agiza, H.N., 1998, "Explicit stability zones for Cournot games with 3 and 4 competitors", Chaos, Solitons \& Fractals 9: 1955-1966.

X Ahmed, E.and Agiza, H.N., 1998, "Dynamics of a Cournot game with $n$ competitors", Chaos, Solitons \& Fractals 9: 1513-1517.

X Bischi, G.-I., Mammana, C,. and Gardini, L., 2000, "Multistability and cyclic attractors in duopoly games", Chaos, Solitons, \& Fractals 11:543-564

X Cuornot, A, 1838, Recherches sur les Principes Mathématiques de la Théorie des Richesses, Hachette, Paris

$\boldsymbol{X}$ Dana, R.-A., and Montrucchio, L., 1986, "Dynamic complexity in duopoly games", Journal of Economic Theory 40: 40-56.

$\boldsymbol{X}$ Edgeworth, F. Y., 1897, "La teoria pura del monopolio", Giornale degli Economisti 15:13-31

X Kuznetsov, Y. A., 1995, Elements of Applied Bifurcation Theory, Springer-Verlag

$\boldsymbol{X}$ Palander, T.F., 1939, "Konkurrens och marknadsjämvikt vid duopol och oligopol", Ekonomisk Tidskrift 41: 124-145 and 222-250.

$X$ Puu, T., 1991, "Chaos in duopoly pricing", Chaos, Solitons \& Fractals 1: 573-581.

$x$ Puu, T., 2000, Attractors, Bifurcations \& Chaos - Nonlinear Perspectives in Economics, Springer-Verlag

$\boldsymbol{x}$ Puu, T. and Suskho, I., 2002, Oligopoly Dynamics - Models and Tools, SpringerVerlag

$\boldsymbol{X}$ Rand, D., 1978, "Exotic phenomena in games and duopoly models", Journal of Mathematical Economics 5: 173-184. 



\section{CERUM Working Papers}

Most of these are available at:

www.umu.se/cerum/publikationer/index.html

1. Einar Holm, Ulf Wiberg (Red.) (1995, in Swedish) Samhällseffekter av Umeå universitet

2. Örjan Pettersson, Lars Olof Persson, Ulf Wiberg (1996, in Swedish) Närbilder av västerbottningar - materiella levnadsvillkor och hälsotillstånd $i$ Västerbottens län

3. Jeanette Edblad (1996) The Political Economy of Regional Integration in Developing Countries

4. Lena Sahlin, Lars Westin (1996, in Swedish) Prissättning av subventionerad kultur. Vilka är de internationella erfarenheterna?

5. Lars Westin, Mats Forsman (1997, in Swedish) Regionerna och finansieringen av infrastrukturen: Exemplet Botniabanan

6. Erik Bergkvist, Lars Westin (1997) Estimation of gravity models by oLs estimation, NLs estimation, Poisson, and Neural Network specifications

7. Niklas Nordman (1998) Increasing Returns to Scale and Benefits to Traffic. A Spatial General Equilibrium Analysis in the Case of Two Primary Inputs

8. Lars Westin, Niklas Nordman (1998) The dialogue of universities with their partners: The case of Umeå University, Sweden

9. Robert Sörensson (1998, in Swedish) Systemanalys av godstransporter. Simulering av en uppgraderad Inlandsbana

10. Carina Sundgren (1998, in Swedish) Beräkning av bruttoregionprodukter för Sveriges regioner. En analys av metodvalet och regionindelningens betydelse

11. Erik Sondell (1999, in Swedish) Halvtidsutvärdering av Interreg-projektet Virtual Education Environment MittSkandia

12. Erik Sondell (1999, in Swedish) Det regionala uppdraget: En fjärde uppgift?

13. Örjan Pettersson (1999) Population Changes in Rural Areas in Northern Sweden 1985-1995

14. Robert Pettersson (1999) Foreign Second Home Purchases: The Case of Northern Sweden, 1990-96

15. Patrik Asplund, Niklas Nordman (1999) Attitudes toward the Third Mission: A Selection of Interviews from Seven Universities in Sweden

16. Kent Eliasson, Magnus Johansson, Lars Westin (1999) European Integration: Eastern Europe and the Swedish Regions

17. Janerik Gidlund, Sverker Sörlin, Susanne Gidlund (2000, in Swedish) Ensam hemma. Den norrländska elitens nya syn på regional utveckling

18. Christine Hudson (2000) The University and Regional Reciprocity

19. Linda Helgesson (2000) Why Some Girls Go to School and Others Don't. A study about girls' education on an upper primary level in northern Mozambique

20. Hans Åkerlind (2000, in Swedish) Framtidens stad

21. Göran Aldskogius (2000) Urban Policy in the Structural Policy of the European Union

22. Leif Kåpe (2000, in Swedish) Förändringar i medelstora svenska städer

23. Örjan Petterson, Anna Nordström, Linda Rislund (2000, in Swedish) Utvärdering av LEADER II Stad och Land - Hand $i$ Hand

24. Sören Olsson (2000, in Swedish) Stadens attraktivitet och det offentliga stadslivet

25. Maria Asplund (2000, in Swedish) Elektronik-och dataingenjörsutbildningen $i$ Pajala, Studentperspektivet

26. Lars Marcus (2000) On Architectural Knowledge

27. Henry Etzkowitz, Patrik Aslund, Niklas Nordman (2001) Beyond Humboldt: Emergence of Academic Entrepreneurship in the U.s. and Sweden 
28. Maria Asplund (2001, in Swedish) Om måluppfyllelsen för Umeå universitets elektronik- och dataingenjörsutbildning i Pajala

29. Maria Asplund, Anna Nordström (2001, in Swedish) Utvärdering av sAms-projektet

30. Eva Bergdahl, Magnus Rönn (2001, in Swedish) Planering för funktionsintegrering - problem och utgångspunkter

31. Maria Asplund (2001, in Swedish) Ex Ante utvärdering av E12 Alliansen

32. Olof Stjernström (red.), Stig-Olof Holm, Johan Håkansson, Urban Lindgren, Håkan Myrlund, Jesper Stage, Kerstin Westin, Lars Westin, Ulf Wiberg (2001, in Swedish) Den hållbara regionen. Om förutsättningar och framtidsmöjligheter för en hållbar samhällsutveckling $i$ Västerbottens län - ett projektförslag

33. Gemma Francès, Ian Layton, Jordi Rosell, Joan Santana, Erik Sondell, Lourdes Viladomiu (2001) The Measurement of On-Farm Diversification

34. Johan Lundberg (2001) On the Determinants of Average Income Growth and Net Migration at the Municipal Level in Sweden

35. Johan Lundberg (2001) A Spatial Interaction of Benefit Spillovers from Locally Provided Public Services

36. Chris Hudson (2001, in Swedish) Regionala partnerskap - ett hot mot eller ett förverkligande av demokrati?

37. Krister Sandberg, Jörgen Johansson (2001) Estimation of Hedonic Prices for Cooperative Flats in the City of Umea with Spatial Autoregressive GMM

38. Elin Lundmark (2002, in Swedish) Fastighetstaxeringsvärdets spridningsmönster i centrala Umeå

39. Ulf Wiberg (2002, in Swedish) Hållbarhet i glesa regionala strukturer-exemplet södra Norrlandskusten

40. Robert Sörensson (2002) Estimation of Interregional Empty Rail Freight Car Flows

41. Emma Lundholm (2002, in Swedish) Den sociala ekonomin i glesa miljöer - en teoretisk diskussion

42. Niklas Bergström (2002, in Swedish) Kontraurbanisering i Umeåregionen

43. Ian Layton, Linda Rislund (2002) Socio-Economic Dimensions of Agricultural Diversification in Västerbotten, Northern Sweden

44. Aurora Pelli (2002) Coping with Innovative On-farm Diversification - a Qualitative Analysis of Farm Household Case Histories from Västerbotten, Sweden

45. Linda Sandberg (2002, in Swedish) Rädslans restriktioner - En studie av kvinnors rädsla i Umeå

46. Martin Paju (2002, in Swedish) Kulturmiljön och den regionala tillväxten - Länsantikvariernas syn på de regionala tillväxtavtalen

47. Tönu Puu, Irina Sushko (2002) A Business Cycle Model with Cubic Nonlinearity

48. Patricia Morton (2002) Social Life and Urban Form in a Historical Perspective

49. (2002)

50. Tönu Puu, Anna Norin (2002) Cournot Duopoly when the Competitors Operate under Capacity Constraints 

The Centre for Regional Science at Umeå University, CERum, initiates and accomplishes research on regional development, carries out multidisciplinary research, and distributes the results to various public organisations. The research projects are pursued in interaction with the numerous scientific disciplines within the regional science field.

The CERUM Working Paper are interim reports presenting work in progress and papers that have been submitted for publication elsewhere. These reports have received only limited review and are primarily used for in-house circulation.

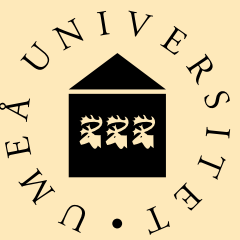

CERUM

CERUM; Umeå University; sE-90187 Umeå; Sweden

Ph.: +46-90-786.6079 Fax: +46-90-786.5121

Email: regional.science@cerum.umu.se

www.umu.se/cerum

ISBN 91-7305-320-1

ISSN $1404-5362$ 